\title{
Accuracy and Reliability of the Optoelectronic Plethysmography and the Heart Rate Systems for Measuring Breathing Rates Compared with the Spirometer.
}

\author{
Laurent Stubbe \\ Ecole Supérieure d'Ostéopathie \\ Nicolas Houel ( $\square$ nicolas.houel@univ-reims.fr) \\ University of Reims Champagne-Ardenne \\ François Cottin \\ University of Paris-Sud
}

\section{Research Article}

Keywords: optoelectronic plethysmography, electrocardiogram, RR variability, breathing rate, spirometer

Posted Date: November 5th, 2021

DOl: https://doi.org/10.21203/rs.3.rs-999985/v1

License: (c) (1) This work is licensed under a Creative Commons Attribution 4.0 International License.

Read Full License 


\section{Abstract}

Measuring breathing rates without a mouthpiece is of interest in clinical settings. Electrocardiogram devices and, more recently, optoelectronic plethysmography (OEP) methods can estimate breathing rates with only a few electrodes or motion-capture markers placed on the patient. This study estimated and compared the accuracy and reliability of three non-invasive devices: an OEP system with 12 markers, an electrocardiogram device and the conventional spirometer. Using the three devices simultaneously, we recorded 72 six-minute epochs on supine subjects. Our results show that the OEP system has a very low limit of agreement and a bias lower than $0.4 \%$ compared with the spirometer, indicating that these devices can be used interchangeably. We observed comparable results for electrocardiogram devices. The OEP system facilitates breathing rate measurements and offers a more complete chest-lung volume analysis that can be easily associated with heart rate analysis without any synchronisation process, for useful features for clinical applications and intensive care.

\section{Introduction}

Various studies have already focused on human physiological rates, including those involving metabolism, hormones, the autonomic nervous system and their interactions ${ }^{1}$. The estimation of human physiological rates is of interest for clinical research and health monitoring with regard to disease prevention ${ }^{2}$. The analysis of these rates help to better understand the interactions between human physiological systems from a macroscopic point of view ${ }^{3}, 4,5,6$. In this context, measuring breathing rates without a mouthpiece is needed to monitor, treat and/or prevent various health conditions such as chronic obstructive pulmonary disease, post-thoracic surgery, ankylosis spondylitis, breathing kinematics in the context of spinal cord injuries ${ }^{7}, 8$ and more recently for pulmonary complications in severe COVID19 cases $^{9}, 10$.

Variation in various human biological rhythms, such as respiration, hearth rate, blood pressure, are currently estimated using non-invasive devices ${ }^{11}$. Spirometry is the standard method for monitoring breathing. For specific pathological care, monitoring the respiration rate without a mouthpiece has become mandatory. Over the past decade, methods based on electrocardiograms (ECGs) and plethysmograms have become accepted for estimate the breathing rate. For clinical monitoring, the global fast Fourier transform algorithm has been recognized for its accuracy and sensitivity in the identification of the main breathing and heart rates ${ }^{11}{ }^{12}$. The usefulness of these two methods for monitoring breathing has been attributed to the relationship between thoracic motion and hearth rate, known as respiratory sinus arrhythmia ${ }^{12}$. Accordingly, optical fibre sensors are increasingly used to asses breathing rates because they can be associated with magnetic resonance imaging ${ }^{13}, 14,15$. However, this technique has a relatively high limit of agreement (LOA) ranging from $\pm 0,45$ to $\pm 2 \mathrm{cpm}{ }^{13}$, 14. Similarly contact ultrasonic sensors monitor breathing in sleep apnoea syndromes ${ }^{16}, 17$ or in conjunction with emotional state such as anger or happiness. This method has a Kappa coefficient of $k=$ 0.38 compared with oronasal flow ${ }^{17}$. Infrared thermography can estimate normal breathing patterns ${ }^{18}$, 
19,20 , because nasal and torso thermal signatures show a high cross-correlation $(r=0.98)^{21}$. Thermal signatures have an LOA of $\pm 0.5 \mathrm{~s}$ compared with inductance plethysmography ${ }^{22}$. Based on variation in chest wall velocity, a triaxial accelerometer indirectly measures breathing and hearth rate variability 23 , and these physiological signals are highly correlated $(r=0.96)$ with those estimated from chest deformation gauges and pulse oximeters ${ }^{24}$. However, accelerometers show time-cumulative errors compared with spirometer measurements ${ }^{25}$, and they have an LOA of $\pm 4 \mathrm{cpm}$ compared with ECGs ${ }^{26}$. Finally, although all these devices are more or less accurate in estimating human breathing rates, they do not provide information on the biomechanics associated with breathing rates.

Structured light plethysmography offers a better estimate of three-dimensional chest wall motion and its frequency ${ }^{27},{ }^{28}$. This marker-less method can be used to reconstruct chest wall movements in clinical applications ${ }^{29},{ }^{30}$. Respiratory volume monitored by structured light plethysmography correlates $\left(R^{2}>\right.$ 0.91 ) with spirometer measurements ${ }^{27}$. However to our knowledge, the accuracy and reliability of structured light plethysmography have not been investigated. Based on infrared cameras, optoelectronic plethysmography (OEP) is a motion-capture method that provides an accurate and reliable threedimensional reconstruction of chest wall movements. The first OEP was developed 30 years ago 31,32 based on video recordings of 32 passive motion-capture markers placed on the subject's torso to measure three-dimensional chest volumes and the variability in chest wall surface motion and to estimate nine chest volumes. In these conditions, the 3D accuracy of the OEP system was SD $=0.06 \mathrm{~mm}$ 31. Increasing the number of markers in the vertical and horizontal planes (some studies have used up to 89 markers) can improve accuracy 32,33 . However, due to its potential clinical applications, OEP was adapted with 24 markers and 9 virtual markers on the subject's back to study breathing in newborns ${ }^{34}$. Recent studies have shown that increasing the number of markers always improved accuracy in OEP. According to ${ }^{35}$, bias and limit of agreement were lower when OEP is associated with 30 markers (bias = $0.056 \mathrm{I}$ and LOA $\pm 0.35 \mathrm{I}$ ) compared with 89 markers (bias $=0.16 \mathrm{I}$ and LOA $\pm 0.4 \mathrm{I}$ ). Moreover, OEP associated with 16 markers seems to be sufficient to monitor tidal volume in spontaneous breathing ${ }^{36}$, and other studies have shown that OEP associated with less than 16 markers can be used to estimate breathing rates and specific biomechanical parameters, such as the ratio between thoracic and abdominal breathing movements (13 markers; Kaneko \& Horie ${ }^{37}$ ) or sternal angle variation (6 markers; Gaillard et al. ${ }^{38}$ ). To our knowledge, only Shafiq \& Veluvolu ${ }^{39}$ have used OEP associated with 16 markers to monitor breathing and cardiac frequencies, simultaneously according to the chest wall marker positions in Alnowan et al. ${ }^{40}$, with 12 of them presenting characteristic signals due to their proximal position on the diaphragm ${ }^{39}$. These 12 markers better predict diaphragm movements using abdominal chest volumes ${ }^{41}$ and provide estimates of breathing rates. Only two studies ${ }^{42}, 34$ have compared the accuracy of OEP compared with the standard method on more than 10 subjects, (10 adults and 20 infants, respectively). Both studies used more than 16 retro-reflexives markers placed on the anterior torso (respectively 45 markers for adults and 24 markers for infants). To our knowledge, no study has assessed the accuracy and reliability of breathing rate estimates from a 12-marker OEP system compared with those from a standard method. 
The aim of the present study was to evaluate the accuracy and reliability of monitoring breathing rates using an OEP system and an ECG device compared with the standard spirometer. We tested whether controlled breathing rates estimated using OEP and ECG can reproduce spirometer measurements with the same accuracy and reliability.

\section{Methods}

\section{Experimental design:}

Here, we estimated breathing rates from an OEP system as well as from ECG signals and heart rate (RR) intervals using an ECG device and compared them with spirometer measurements during audiocontrolled subject breathing.

\section{Subjects:}

Twenty-nine volunteers participated in the present study. Subjects ( 17 females and 12 males; mean \pm standard deviation $(\mathrm{SD})$ : age $=19.69 \pm 1$ years; height $=170.45 \pm 9.59 \mathrm{~cm}$; body mass $=61.79 \pm 13.41$ $\mathrm{kg})$. The study was approved by by the local ethics committee at the Ecole Superieure d'Osteopathie Paris (France) and carried out in accordance with the Declaration of Helsinki (World Medical Declaration of Helsinki, 2013). All subjects were informed of the objectives of the study and signed the informed consent form before participating.

\section{Experimental setup}

The study took place in a well-ventilated, quiet room with constant temperature $\left(=22^{\circ} \mathrm{C}\right)$, without ultraviolet disturbances. Subjects were instructed to refrain from smoking, and to avoid caffeine and other stimulants (medication or drugs) 72 hours before the experiment ${ }^{43}$. Each subject was greeted and invited to relax without any stimulation in a resting room for 30 minutes according to the task force recommendations ${ }^{2}$. Then, the subject lay down in supine position on a large table to maximise relaxation and limit any stress associated with physiological monitoring. The subject was instructed to inhale and exhale at a frequency of $0.25 \mathrm{~Hz}$ in rhythm with an audio recording for $7 \mathrm{~min}$ followed by one breath at maximal tidal volume ${ }^{43}, 44$. The subject performed the whole breathing exercise (audio-guided breathing and one maximal tidal volume breath) three times separated by a seven-minute recovery period. During the whole breathing exercise, physiological parameters were recorded using an OEP system, an ECG and a spirometer. A spirometer was mounted on a hands-free support (Figure 1) to limit discomfort and restraints (cf. Niérat et al. ${ }^{45}$ ).

\section{OEP procedure}


Twelve retro-reflexive markers (12 $\mathrm{mm}$ each) were placed on the chest (Figure 2), in accordance with previous studies $31,41,40,39$. Markers were distributed on the surface of the chest wall at the 12 intersections of six planes: a median coronal plane crossing the chest, two sagittal medial-clavicular planes, a transverse subxiphoid plane, a transverse subcostal plane and a median transverse plane between the two previous transverse planes (see Figure 3). For recording, three other markers were placed on the table to define a reference plane ${ }^{33}, 42,34$.

Chest marker positions were recorded with eight infrared cameras (MXT10) at a $100 \mathrm{~Hz}$ sampling frequency. Cameras were time-synchronized using an Vicon Nexus 1.8.5 optoelectronic system and with an MX Giganet link ${ }^{46}$. Standard deviation of the reference plane ${ }^{31}$ was equal to $0.062 \mathrm{~mm}$.

\section{Spirometer and ECG procedures}

During each session, breathing was continuously recorded using a spirometer. Cardiovascular signals (ECG) were also continuously recorded at $1000 \mathrm{~Hz}$. RR-interval time series were extracted from raw ECG signals ${ }^{47}$. Respiration and cardiovascular signals were recorded, digitalized and synchronized using a Power-Lab 8/35 device (Human respiratory kit with spirometer and ECG Bio Amps, ADInstrument ${ }^{\circledR}$ ). Calibrations were performed according to the manufacturer's instructions before each test on each subject.

\section{Synchronization setup}

Kinematics, ECG and spirometer data were time-synchronized by determining the minimum local signal during the exhale at tidal maximal volume, similar to the procedure in Lo Presti et al. ${ }^{48}$. For each signal, a six-minute epoch before the synchronization point was selected. A fast Fourier transform was performed on each signal included in the six-minute epoch.

\section{OEP processing}

Chest volume was first computed using the 12 subject markers and the three reference plane markers. The projection of the position of the 12 markers was calculated in the reference plane. Six volumes were computed using the parallelepiped equation (Figure 3). Chest volume was equal to the sum of the six previous parallelepipeds. Fast Fourier transform was performed on the chest volume signal for each sixminute epoch. The local maximum amplitude frequency signal nearest to $0.25 \mathrm{~Hz}$ was extracted (Figure 4a). This frequency matches the subject's own breathing rate during the controlled breathing exercise.

\section{Physiological data processing}


For each physiological data point (spirometer, ECG and RR series), the fast Fourier transform was applied to the same six-minute epoch used for OEP recordings. The same local maximal amplitude frequency signal nearest to $0.25 \mathrm{~Hz}$ was extracted (Figure $4 \mathrm{~b}, \mathrm{c}, \mathrm{d}$ ). All data processing was carried out using MATLAB® 2018.

\section{Statistical analysis}

The mean and standard deviation of breathing rates were computed. Reliability and agreement between OEP and ECG estimations were compared with spirometer data according to Kottner et al. (2011) recommendations. Correlation coefficients $(r)$ were used to estimate the relationship between breathing rate data from OEP, ECG and RR with spirometer measurements. Testing the mean against a constant reference value was done to compare OEP as well as ECG and RR with spirometer values. The threshold alpha value was set to 0.05 . A Bland-Altman plot was used to define accuracy and reliability between the OEP- ECG- or RR-based and spirometer breathing rates ${ }^{50}, 51$.

\section{Results}

In all, 81 recordings were taken with the three devices (OEP, ECG (ECG and RR signals), spirometer). Nine records were excluded from the OEP data due to insufficient or absence of maximal tidal volume, thereby limiting the synchronization process. Ultimately, 72 simultaneous recordings were analysed based on OEP signals and spirometer measurements and 81 simultaneous recordings were analysed based on ECG signals and spirometer measurements.

\section{OEP versus spirometer analysis}

The mean $( \pm$ SD) OEP and spirometer breathing rates were respectively equal to $0.2472 \pm 0 \mathrm{~Hz}$ and 0.248 $\pm 0 \mathrm{~Hz}$. Correlation coefficients showed a high values $(r=1, p<0.001)$. The Bland-Altman plot indicated a significant bias equal to $8 \times 10^{-4} \mathrm{~Hz}$ and an LOA of $\pm 0 \mathrm{~Hz}$ (Figure 5). Figures $4 \mathrm{a}$ and $4 \mathrm{~b}$ showed that breathing rates estimated using OEP signals were as strong as those based on spirometer signals due to the high amplitude value in the frequency domain. Heart rates estimated from the OEP signal (around $0.74 \mathrm{~Hz}$ ) showed a lower amplitude than the heart rate signal based on spirometer and RR data.

\section{ECG versus spirometer analysis}

The mean ( \pm SD) ECG and spirometer breathing rates were respectively equal to $0.2477 \pm 0.0019 \mathrm{~Hz}$ and $0.248 \pm 0 \mathrm{~Hz}$. Testing the mean against the constant reference value showed no significant differences between ECG and spirometer data $(p=0.195)$. Correlation coefficients were high $(r=1, p<0.001)$. A Bland-Altman plot showed a significative bias equal to $2.89 \times 10^{-4} \mathrm{~Hz}$ and an LOA of $\pm 3.91 \times 10^{-3} \mathrm{~Hz}$ (Figure 6). Figures $4 \mathrm{c}$ and $4 \mathrm{~b}$ show that breathing rates estimated from ECG signals had a lower amplitude than the breathing signal given by the spirometer in the frequency domain. However, the heart 
rate estimated from the ECG signal (around $0.74 \mathrm{~Hz}$ ) showed a greater amplitude than the heart rate signal from the spirometer.

\section{RR versus spirometer analysis}

The mean ( \pm SD) RR and spirometer breathing rates were respectively equal to $0.248 \pm 0.0013 \mathrm{~Hz}$ and $0.248 \pm 0 \mathrm{~Hz}$. Testing the mean against the constant reference value showed no significant difference between RR and spirometer data $(p=0.537)$. The correlation coefficient was high $(r=1, p<0.001)$. The Bland-Altman plot showed a significant bias equal to $-9.26 \times 10^{-5} \mathrm{~Hz}$ and an LOA of $\pm 2.64 \times 10^{-3} \mathrm{~Hz}$ (Figure 7). Figures $4 d$ and $4 \mathrm{~b}$ show that breathing rates estimated from the RR signal were as strong as breathing signals from the spirometer in the frequency domain. Heart rates estimated based on the RR signal (around $0.74 \mathrm{~Hz}$ ) were as strong as heart rates based on spirometer data.

\section{Discussion}

The aim of the present study was to estimate the accuracy and reliability of breathing rates estimated using an OEP system or an ECG compared with those measured using the standard spirometer method. The results of the present study showed that breathing rates estimated from an OEP system based on only 12 motion-capture markers or from ECG closely corroborate spirometer-based measurements.

Agreement between OEP analysis and spirometer measurements

Breathing rates estimated using an OEP system were in agreement with spirometer recordings taken following Kottner et al. ${ }^{49}$ recommendations, with an LOA of $\pm 0 \mathrm{~Hz}$ and a bias of $8 \times 10^{-4} \mathrm{~Hz}$. This bias represents $0.32 \%$ of the reference value. Previous studies have already shown the accuracy and reliability of kinematics analysis for estimating chest volume $32,34,31$. However, to our knowledge, the results of previous studies were based on 45 markers and measurements on fewer than 10 adults. The improved of OEP estimates of breathing rates can be attributed to our calibration process that provides a spacevolume reconstruction with a accuracy of $\pm 0.062 \mathrm{~mm}$. This level of accuracy has been already observed in other studies on chest volume ${ }^{31}$ and spinal curve ${ }^{46}$. The main advantage of OEP analysis is to provide non-invasive biomedical estimates of chest-wall compartments and therefore variations in lung volume ${ }^{7}, 8$, particularly for mechanically ventilated patients ${ }^{33}$, monitoring breathing in preterm and term infants ${ }^{52}$, or pulmonary complications in severe COVID-19 cases ${ }^{9},{ }^{10}$. In particular, OEP analysis provides simultaneous estimates of breathing and heart rates without a synchronization process and without the stress associated with the use of a mouthpiece, as usually observed with the spirometer device 4845 .

Agreement between ECG (ECG, RR interval) and spirometer recordings

Breathing rates have already been estimated using ECG (ECG signals and RR intervals) recordings (Charlton et al, 2018) ${ }^{53}$. Breathing rates estimated from the ECG signal were in agreement with 
spirometer measurements with an LOA of $\pm 3.91 \times 10^{-3} \mathrm{~Hz}$ and a bias equal to $2.89 \times 10^{-4} \mathrm{~Hz}$. The correlation between both devices was very high. Breathing rates estimated from an RR-interval analysis is in agreement with spirometer measurements with an LOA of $\pm 2.64 \times 10^{-3} \mathrm{~Hz}$ and a bias equal to $-9.26 \times$ $10^{-5} \mathrm{~Hz}$. Biases for ECG and RR were respectively $0.12 \%$ and $0.04 \%$ of the reference value. The correlation of the values from both devices was also very high. No significant differences were observed between ECG signals (ECG, RR intervals) and spirometer data. These results are in agreement with previous studies that showed that breathing rate interacts with heart rate and the RR interval (Charlton et al, 2018) 54,55 . Moreover, breathing rates are usually recorded during heart rate monitoring sessions that use ECGs (ECG signals, RR intervals) ${ }^{53}, 54$. Our results indicate that breathing rates estimated using an RR analysis show less bias and a better LOA than ECG analysis. Moreover, Figure 4 demonstrates that breathing rates were more easily detected in the frequency domain using RR-interval analysis. These results confirm the advantage of performing RR analysis to better monitor and diagnose heart rate and breathing variability compared with the usual clinical analysis based on the time and the frequency domains ${ }^{53}, 11$.

Both OEP- and ECG-based analyses show accuracy and reliability on par with the spirometer. Due to its negligible LOA and its bias lower than 0.4\%, OEP analysis offers more opportunities for biomedical monitoring than ECG devices. OEP signals provide higher power of detection of breathing frequency and more opportunities to define chest lung volume and the breathing rate.

The main limitation in the present study was the use of audio recordings to guide the subject's breathing. Audio recordings has been already used in previous studies to better estimate both breathing and heart rate $56,54,55$. Here audio recordings were to define breathing and heart rate rhythms more easily without complex algorithms other than the fast Fourier transform. Complementary signal processing can be used to improve the estimates of breathing frequency and heart rate variability according to the clinical purposes or to define consensus conditions ${ }^{2}$.

\section{Conclusion}

The present study showed that OEP, and ECG devices can be used interchangeably with respect to the standard spirometer when breathing frequency monitoring is required. All devices showed very low LOA values and bias lower than $1 \%$ compared with the standard spirometer method. The OEP system offers many possibilities to estimate biomedical signals simultaneously (e.g. breathing and heart rates). Similarly to ECG and RR-interval analysis, OEP opens the way for new monitoring solutions associated with specific clinical applications. The non-invasiveness of the kinematics device (only passive markers are placed on the subject's chest) is particularly attractive feature for intensive care and preterm infant care.

\section{Declarations}

\section{Acknowledgements:}


The authors thank the personnel at the ESO Paris research laboratory for their help during this study. We would especially like to thank Roger, Christophe and Olivier Caporossi, Serge Pin and Jeanine Sanchez. We are also grateful to Stéphanie Barocco Lecca for her help during this project. The authors also thank Carolyn Engel-Gautier, science editor, for improving the English usage in the present manuscript and Mickaël Soudain-Pineau for his help with the statistical analysis.

\section{Reference}

1. Goldbeter, A., Gérard, C. \& Leloup, J.-C. Biologie des systèmes et rythmes cellulaires. Med Sci (Paris) 26, 49-56 (2010).

2. Malik, M. et al. Heart rate variability. Standards of measurement, physiological interpretation, and clinical use. Task Force of the European Society of Cardiology and the North American Society of Pacing and Electrophysiology. Eur. Heart J. 17, 354-381 (1996).

3. de Rosnay, J. Le macroscope: Vers une version globale. Infoscience https://infoscience.epfl.ch/record/51502 (1975).

4. Stefanovska, A., Bracic, M. \& Kvernmo, H. D. Wavelet analysis of oscillations in the peripheral blood circulation measured by laser Doppler technique. IEEE Transactions on Biomedical Engineering 46, 1230-1239 (1999).

5. McCraty, R. \& Zayas, M. A. Cardiac coherence, self-regulation, autonomic stability, and psychosocial well-being. Front Psycho/ 5, (2014).

6. Kelso, J. A. S. Coordination Dynamics. in Encyclopedia of Complexity and Systems Science (ed. Meyers, R. A.) 1-41 (Springer, 2009). doi:10.1007/978-3-642-27737-5_101-3.

7. Massaroni, C. et al. Optoelectronic Plethysmography in Clinical Practice and Research: A Review. Respiration 93, 339-354 (2017a).

8. Parreira, V. F. et al. Optoelectronic plethysmography: a review of the literature. Rev Bras Fisioter 16, 439-453 (2012).

9. Daher, A. et al. Follow up of patients with severe coronavirus disease 2019 (COVID-19): Pulmonary and extrapulmonary disease sequelae. Respir Med 174, 106197 (2020).

10. Liu, K. et al. Respiratory rehabilitation in elderly patients with COVID-19: A randomized controlled study. Complement Ther Clin Pract 39, 101166 (2020).

11. Novak, V. et al. Influence of respiration on heart rate and blood pressure fluctuations. J. Appl. Physiol. 74, 617-626 (1993).

12. Charlton, P. H. et al. Breathing Rate Estimation From the Electrocardiogram and Photoplethysmogram: A Review. IEEE Reviews in Biomedical Engineering 11, 2-20 (2018).

13. Dziuda, Ł., Skibniewski, F. W., Krej, M. \& Baran, P. M. Fiber Bragg grating-based sensor for monitoring respiration and heart activity during magnetic resonance imaging examinations. J Biomed Opt 18, 57006 (2013). 
14. Lau, D. et al. Intensity-modulated microbend fiber optic sensor for respiratory monitoring and gating during MRI. IEEE Trans Biomed Eng 60, 2655-2662 (2013).

15. Yoo, W.-J. et al. Development of Respiration Sensors Using Plastic Optical Fiber for Respiratory Monitoring Inside MRI System. J. Opt. Soc. Korea, JOSK 14, 235-239 (2010).

16. Arlotto, P., Grimaldi, M., Naeck, R. \& Ginoux, J.-M. An ultrasonic contactless sensor for breathing monitoring. Sensors (Basel) 14, 15371-15386 (2014).

17. De Chazal, P. et al. Sleep/wake measurement using a non-contact biomotion sensor. J Sleep Res 20, 356-366 (2011).

18. Cardone, D., Pinti, P. \& Merla, A. Thermal Infrared Imaging-Based Computational Psychophysiology for Psychometrics. Comput Math Methods Med 2015, 984353 (2015).

19. Murthy, R. \& Pavlidis, I. Noncontact measurement of breathing function. IEEE Eng Med Biol Mag 25, 57-67 (2006).

20. Murthy, J. N. et al. Thermal infrared imaging: a novel method to monitor airflow during polysomnography. Sleep 32, 1521-1527 (2009).

21. Goldman, L. J. Nasal airflow and thoracoabdominal motion in children using infrared thermographic video processing. Pediatr. Pulmonol. 47, 476-486 (2012).

22. Lewis, G. F., Gatto, R. G. \& Porges, S. W. A novel method for extracting respiration rate and relative tidal volume from infrared thermography. Psychophysiology 48, 877-887 (2011).

23. Inan, O. T. Recent advances in cardiovascular monitoring using ballistocardiography. in 2012 Annual International Conference of the IEEE Engineering in Medicine and Biology Society 5038-5041 (2012). doi:10.1109/EMBC.2012.6347125.

24. Ferrer-Lluis, I., Castillo-Escario, Y., Montserrat, J. M. \& Jané, R. Analysis of Smartphone Triaxial Accelerometry for Monitoring Sleep-Disordered Breathing and Sleep Position at Home. IEEE Access 8 , 71231-71244 (2020).

25. Fekr, A. R., Janidarmian, M., Radecka, K. \& Zilic, Z. A medical cloud-based platform for respiration rate measurement and hierarchical classification of breath disorders. Sensors (Basel) 14, 1120411224 (2014).

26. Chan, A. M., Ferdosi, N. \& Narasimhan, R. Ambulatory respiratory rate detection using ECG and a triaxial accelerometer. in 2013 35th Annual International Conference of the IEEE Engineering in Medicine and Biology Society (EMBC) 4058-4061 (2013). doi:10.1109/EMBC.2013.6610436.

27. Gourlay, A. R., Kaye, G., Denison, D. M., Peacock, A. J. \& Morgan, M. D. Analysis of an optical mapping technique for lung function studies. Comput. Biol. Med. 14, 47-58 (1984).

28. Lewis, J. R. T. \& Sopwith, T. Measuring the human chest with structured lighting. Pattern Recognition Letters 4, 359-366 (1986).

29. Elshafie, G. et al. Measuring changes in chest wall motion after lung resection using structured light plethysmography: a feasibility study. Interact Cardiovasc Thorac Surg 23, 544-547 (2016). 
30. Ghezzi, M., Tenero, L., Piazza, M., Bodini, A. \& Piacentini, G. Structured Light Plethysmography (SLP): Management and follow up of a paediatric patient with pneumonia. Respir Med Case Rep 22, 67-69 (2017).

31. Ferrigno, G. et al. Three-dimensional optical analysis of chest wall motion. J. Appl. Physiol. 77, 1224-1231 (1994).

32. Cala, S. J. et al. Chest wall and lung volume estimation by optical reflectance motion analysis. J. Appl. Physiol. 81, 2680-2689 (1996).

33. Aliverti, A. et al. Optoelectronic plethysmography in intensive care patients. Am. J. Respir. Crit. Care Med. 161, 1546-1552 (2000).

34. Dellaca', R. L. et al. Measurement of total and compartmental lung volume changes in newborns by optoelectronic plethysmography. Pediatr. Res. 67, 11-16 (2010).

35. Massaroni, C. et al. Assessment of breathing volumes and kinematics by motion capture systems: Comparison of protocols. in 2017 IEEE International Instrumentation and Measurement Technology Conference (I2MTC) 1-6 (2017c). doi:10.1109/I2MTC.2017.7969817.

36. Laufer, B. et al. Minimum Number of Sensors in a Smart Shirt to Measure Tidal Volumes. IFACPapersOnLine 51, 92-97 (2018).

37. Kaneko, H. \& Horie, J. Breathing movements of the chest and abdominal wall in healthy subjects. Respir Care 57, 1442-1451 (2012).

38. Gaillard, L., Debraux, L. \& Houel, N. Manubrio-sternal joint mobility during forced ventilation using non-invasive opto-electronic plethysmography: cases studies. Computer Methods in Biomechanics and Biomedical Engineering 22, S411-S412 (2019).

39. Shafiq, G. \& Veluvolu, K. C. Multimodal chest surface motion data for respiratory and cardiovascular monitoring applications. Sci Data 4, 170052 (2017).

40. Alnowam, M. R., Lewis, E., Wells, K. \& Guy, M. Respiratory motion modelling and prediction using probability density estimation. in IEEE Nuclear Science Symposuim Medical Imaging Conference 2465-2469 (2010). doi:10.1109/NSSMIC.2010.5874231.

41. Wang, H.-K. et al. Relationship between chest wall motion and diaphragmatic excursion in healthy adults in supine position. J. Formos. Med. Assoc. 108, 577-586 (2009).

42. Aliverti, A. et al. Compartmental analysis of breathing in the supine and prone positions by optoelectronic plethysmography. Ann Biomed Eng 29, 60-70 (2001).

43. Younes, M., Nowakowski, K., Didier-Laurent, B., Gombert, M. \& Cottin, F. Effect of spinal manipulative treatment on cardiovascular autonomic control in patients with acute low back pain. Chiropr Man Therap 25, 33 (2017).

44. Monti, A., Médigue, C. \& Mangin, L. Instantaneous parameter estimation in cardiovascular time series by harmonic and time-frequency analysis. IEEE Trans Biomed Eng 49, 1547-1556 (2002).

45. Niérat, M.-C. et al. Measuring Ventilatory Activity with Structured Light Plethysmography (SLP) Reduces Instrumental Observer Effect and Preserves Tidal Breathing Variability in Healthy and COPD. 
Front Physio/ 8, 316 (2017).

46. Boulet, S., Boudot, E. \& Houel, N. Relationships between each part of the spinal curves and upright posture using Multiple stepwise linear regression analysis. J Biomech 49, 1149-1155 (2016).

47. Van de Louw, A., Médigue, C., Papelier, Y. \& Cottin, F. Breathing cardiovascular variability and baroreflex in mechanically ventilated patients. Am J Physiol Regul Integr Comp Physiol 295, R19341940 (2008).

48. Lo Presti, D. et al. Cardio-Respiratory Monitoring in Archery Using a Smart Textile Based on Flexible Fiber Bragg Grating Sensors. Sensors (Basel) 19, (2019).

49. Kottner, J. et al. Guidelines for Reporting Reliability and Agreement Studies (GRRAS) were proposed. International Journal of Nursing Studies 48, 661-671 (2011).

50. Bland, J. M. \& Altman, D. G. Statistical methods for assessing agreement between two methods of clinical measurement. Lancet 1, 307-310 (1986).

51. Bland, J. M. \& Altman, D. G. Comparing methods of measurement: why plotting difference against standard method is misleading. The Lancet 346, 1085-1087 (1995).

52. Pickerd, N., Williams, E. M. \& Kotecha, S. Electromagnetic inductance plethysmography to measure tidal breathing in preterm and term infants. Pediatr Pulmonol 48, 160-167 (2013).

53. Eckberg, D. L. Physiological basis for human autonomic rhythms. Ann. Med. 32, 341-349 (2000).

54. Beda, A., Simpson, D. M., Carvalho, N. C. \& Carvalho, A. R. S. Low-frequency heart rate variability is related to the breath-to-breath variability in the respiratory pattern. Psychophysiology 51, 197-205 (2014).

55. Brown, T. E., Beightol, L. A., Koh, J. \& Eckberg, D. L. Important influence of respiration on human R-R interval power spectra is largely ignored. Journal of Applied Physiology 75, 2310-2317 (1993).

56. Saboul, D., Pialoux, V. \& Hautier, C. The breathing effect of the LF/HF ratio in the heart rate variability measurements of athletes. Eur J Sport Sci 14 Suppl 1, S282-288 (2014).

\section{Figures}




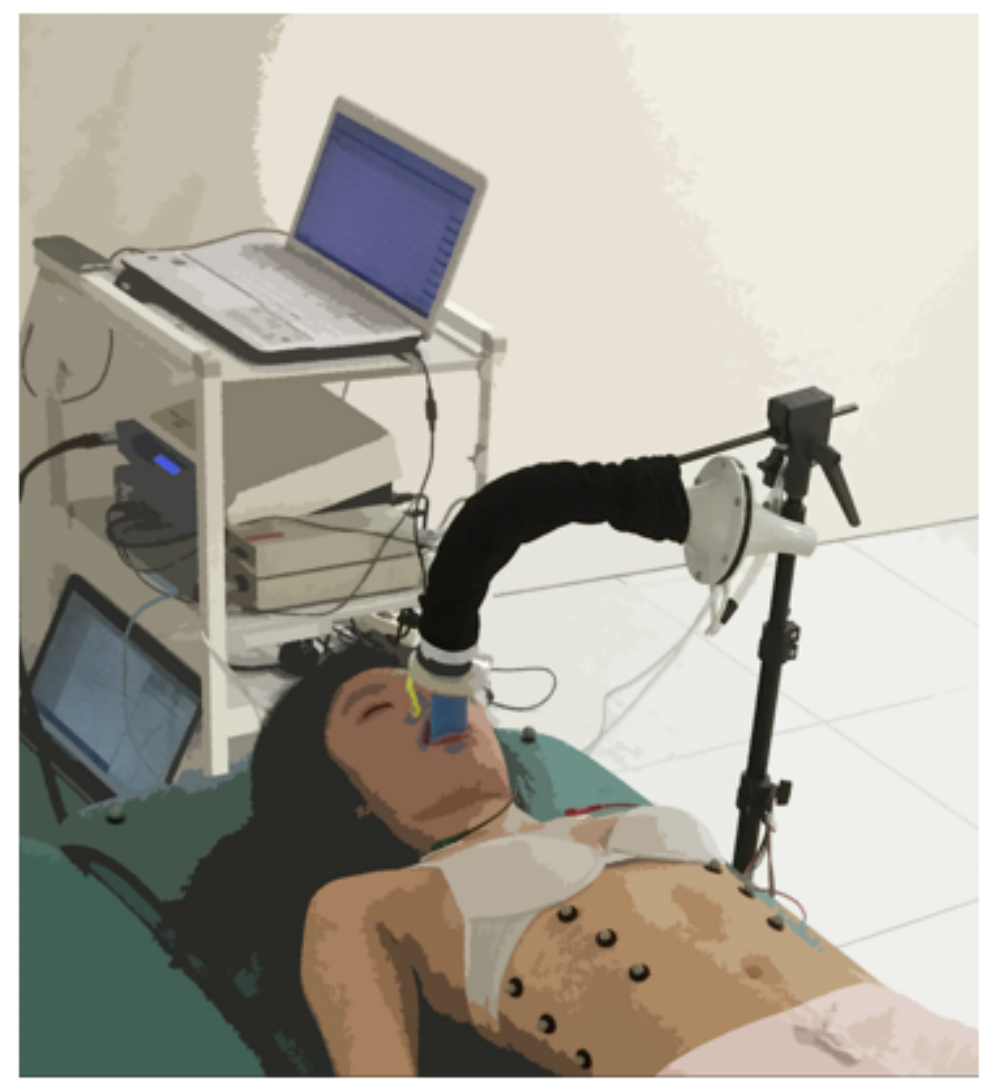

\section{Figure 1}

Subject in a supine position with spirometer mounted on a support to limit discomfort and stress.

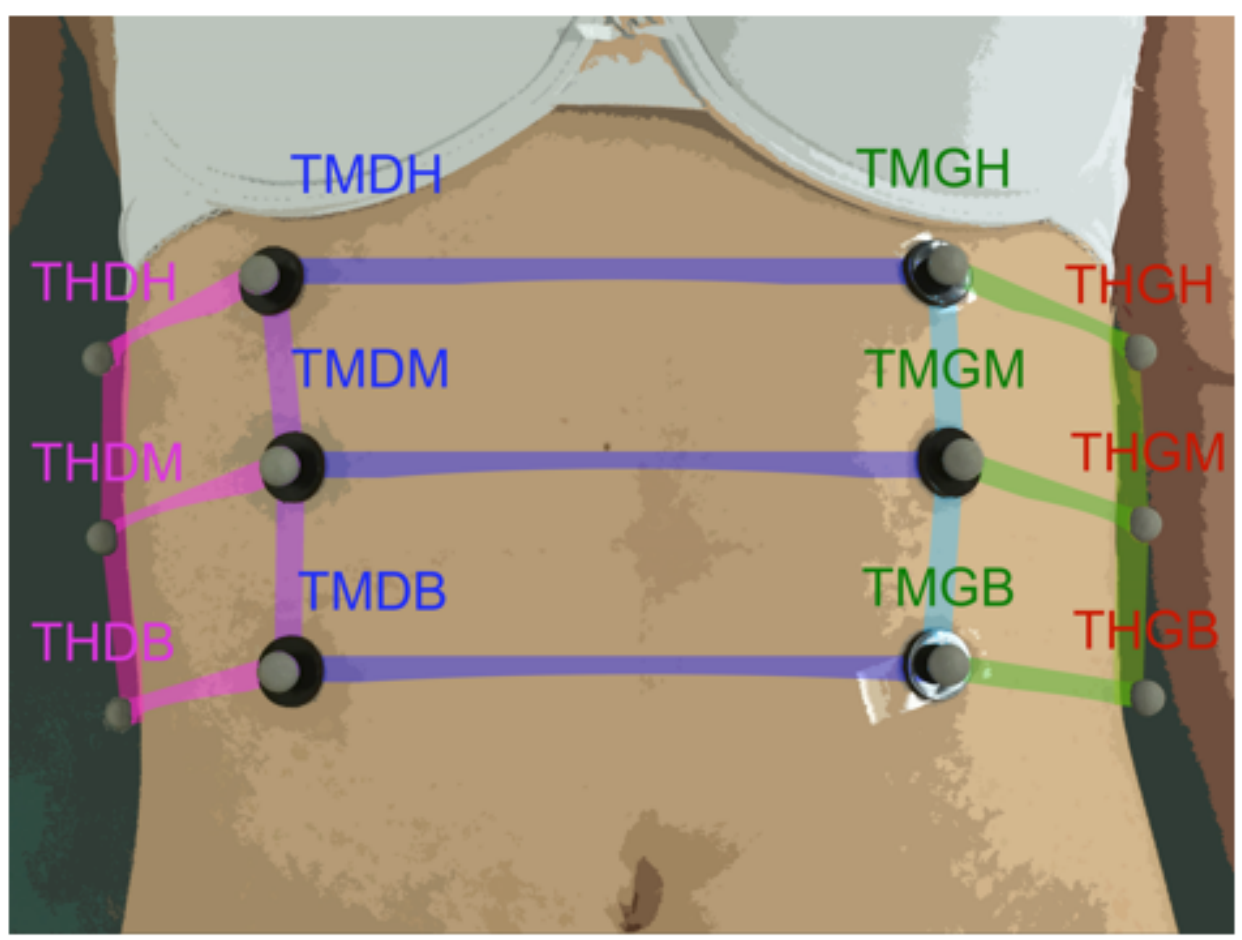

Figure 2 
The 12 retro-reflexive marker positions on the subject at the intersections of six planes on the surface of the chest wall.

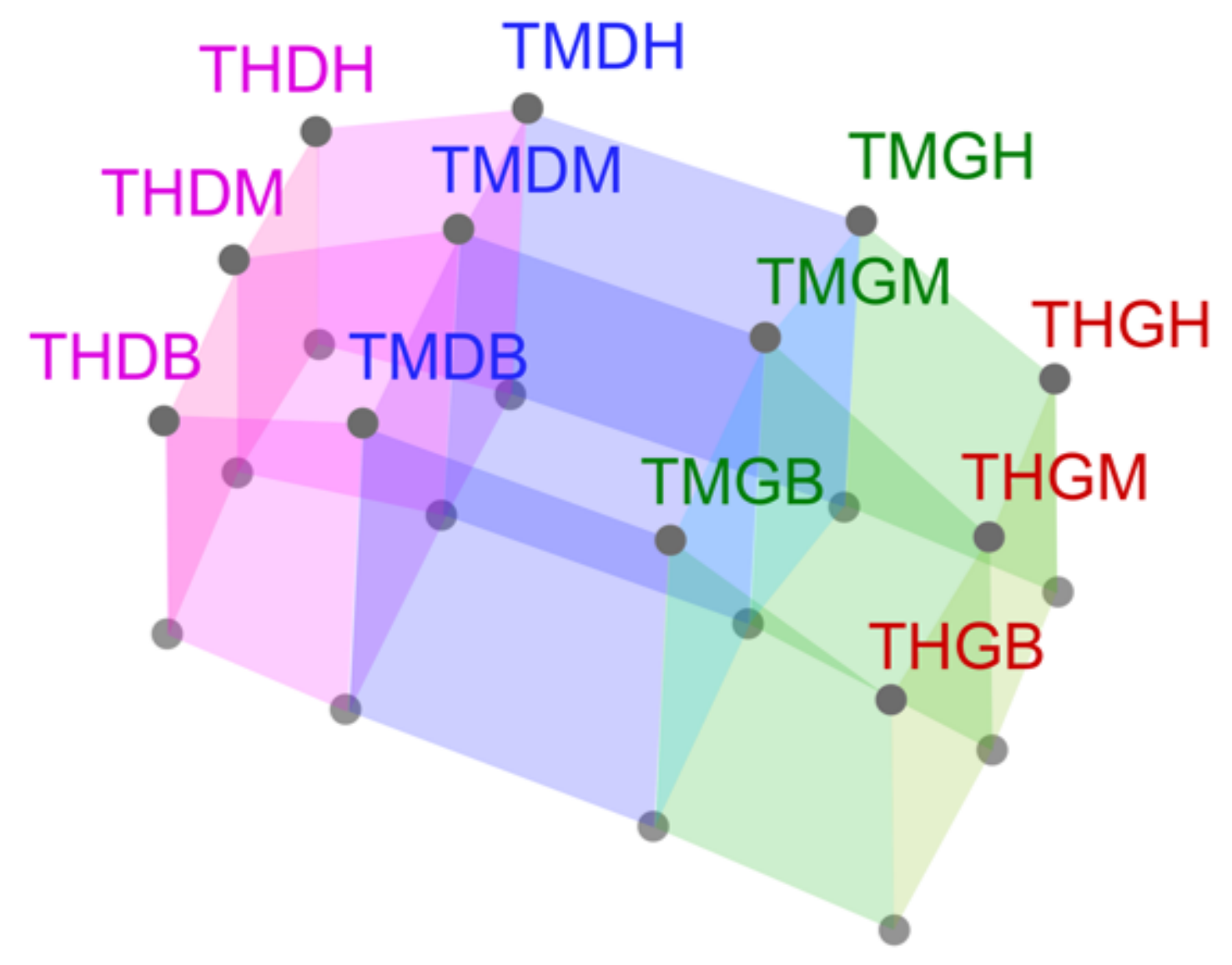

Figure 3

Plot of chest volume computed using six parallelepipeds. Each parallelepiped includes subject markers and its projection in the reference plane. 


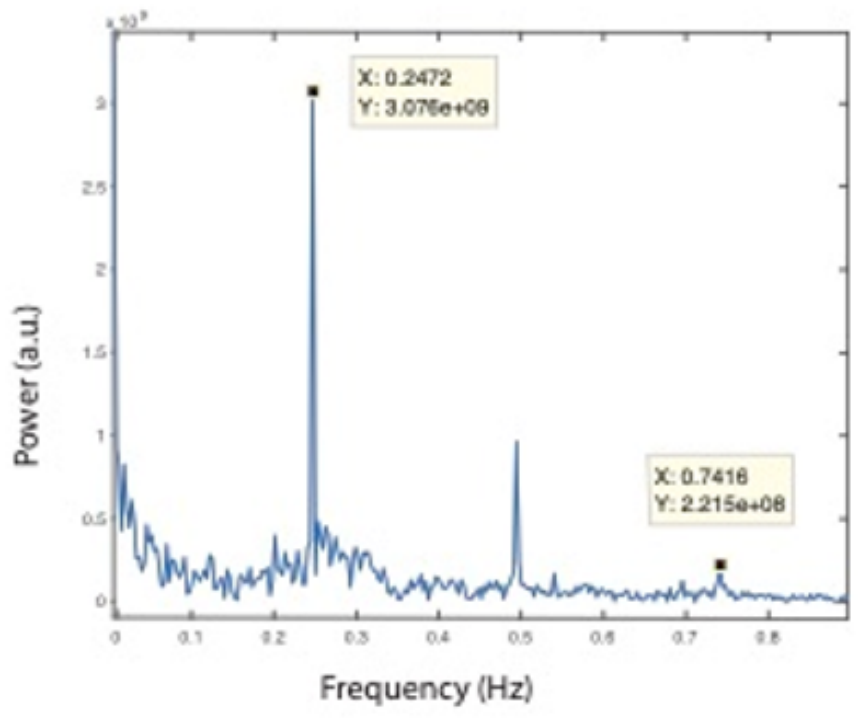

Fig - 4a : FFT OEP signal

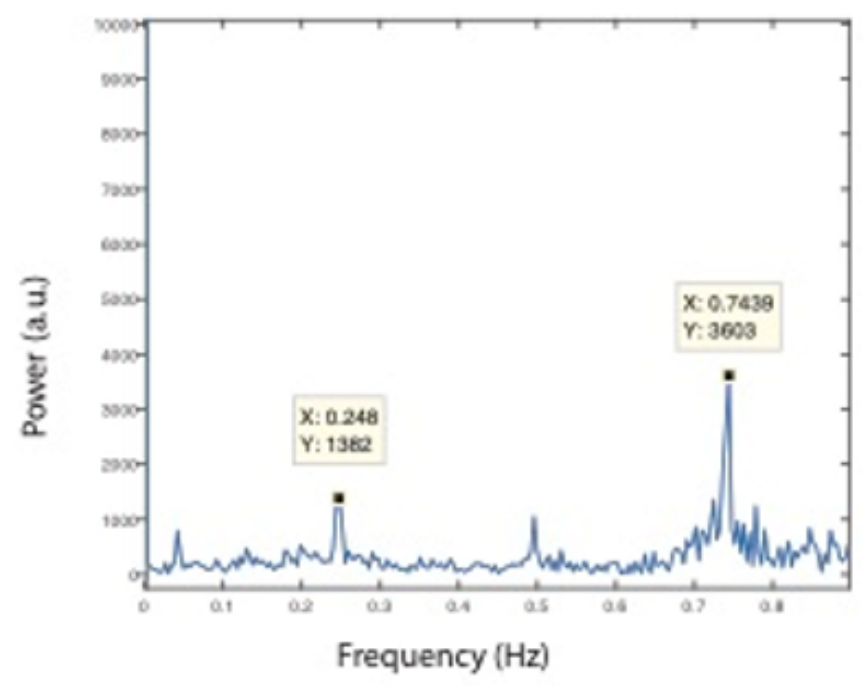

Fig - 4c: FFT ECG signal

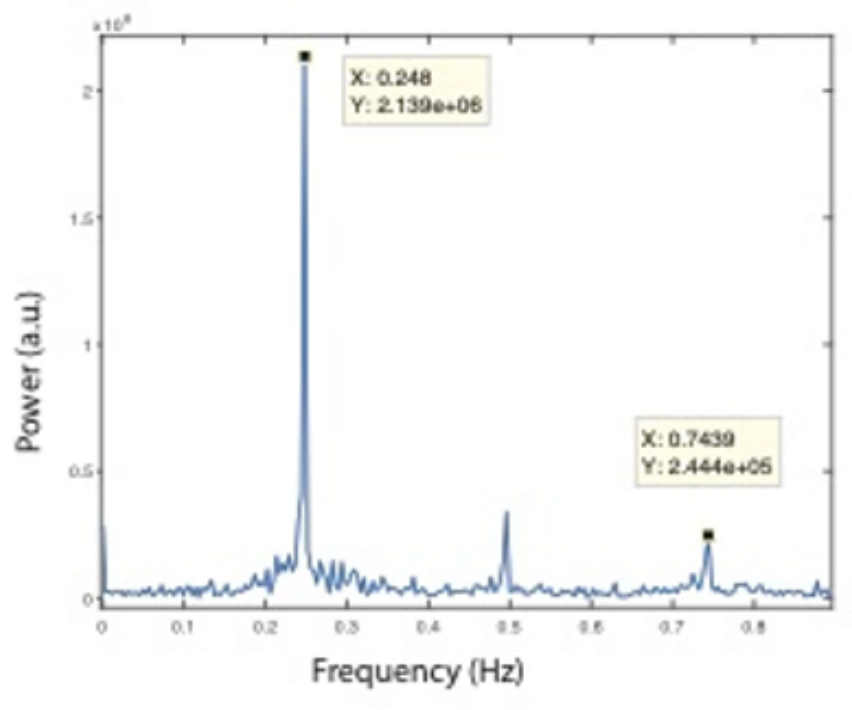

Fig - 4b : FFT spirometer signal

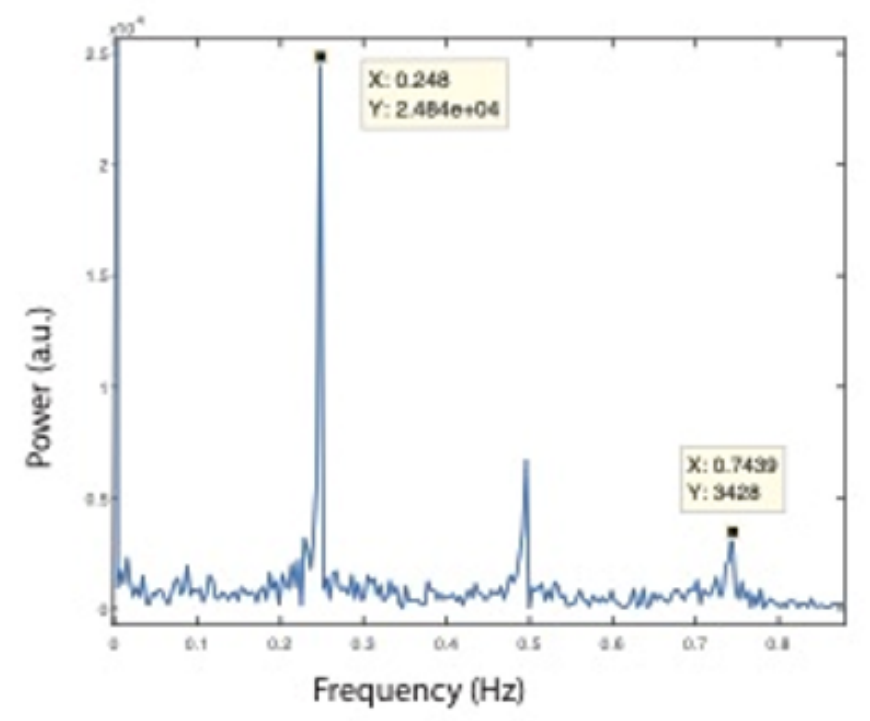

Fig - 4d : FFT RR signal

\section{Figure 4}

Plots of subjects' breathing frequencies nearest to $0.25 \mathrm{~Hz}$ during the controlled six-minute breathing exercise performed using a fast Fourier transform. a) OEP signal (frequency $=0.2472 \mathrm{~Hz}$ ); b) spirometer data (frequency $=0.248 \mathrm{~Hz}$ ); ) ECG data (frequency $=0.248 \mathrm{~Hz}$ ) and d) with RR-interval time series (frequency $=0.248 \mathrm{~Hz}$ ). 


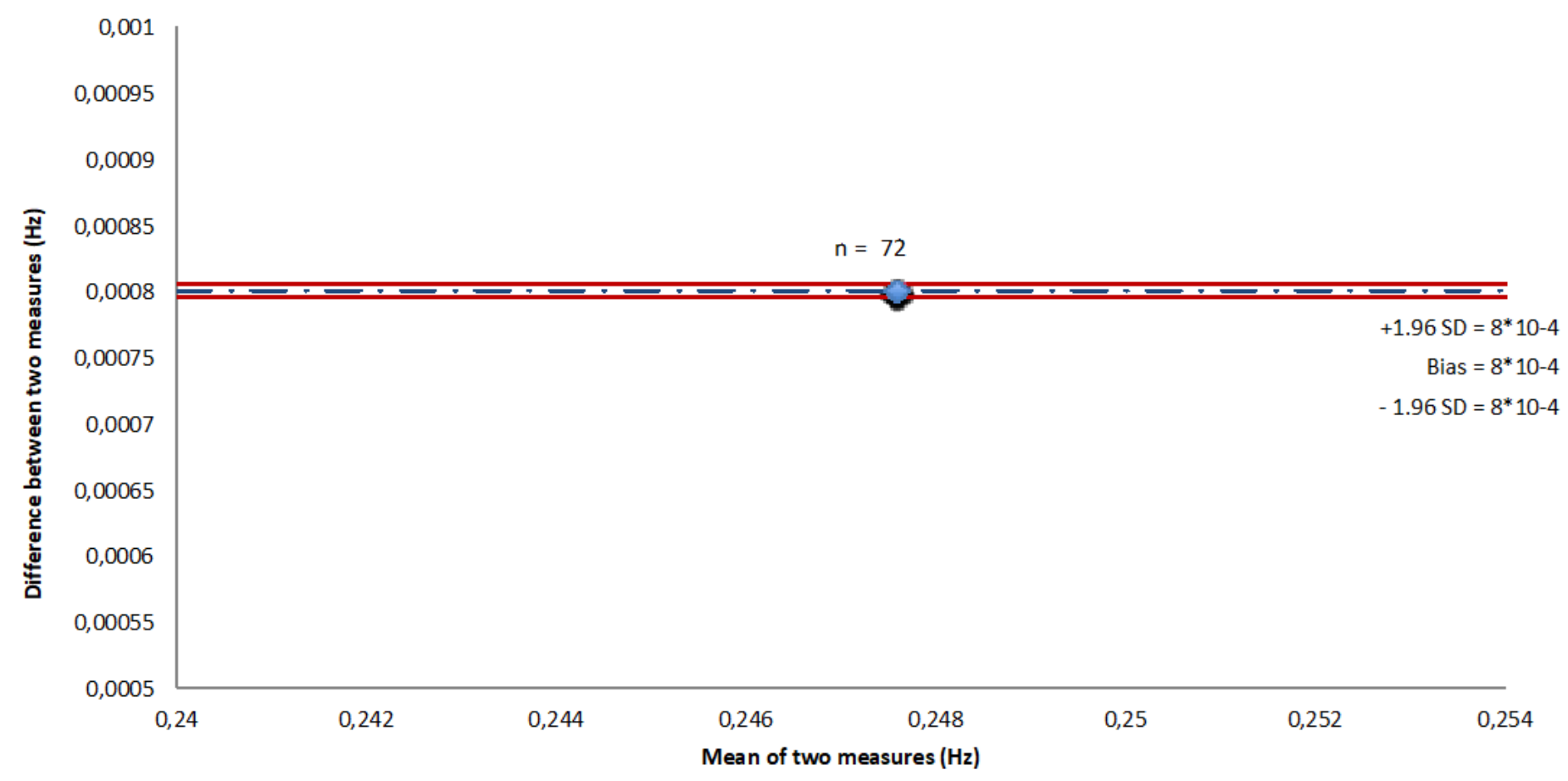

\section{Figure 5}

Plot of Bland-Altman showing the bias (broken line) and $95 \%$ limits of agreement (continuous line) between OEP chest volume frequencies and spirometer breathing rates.

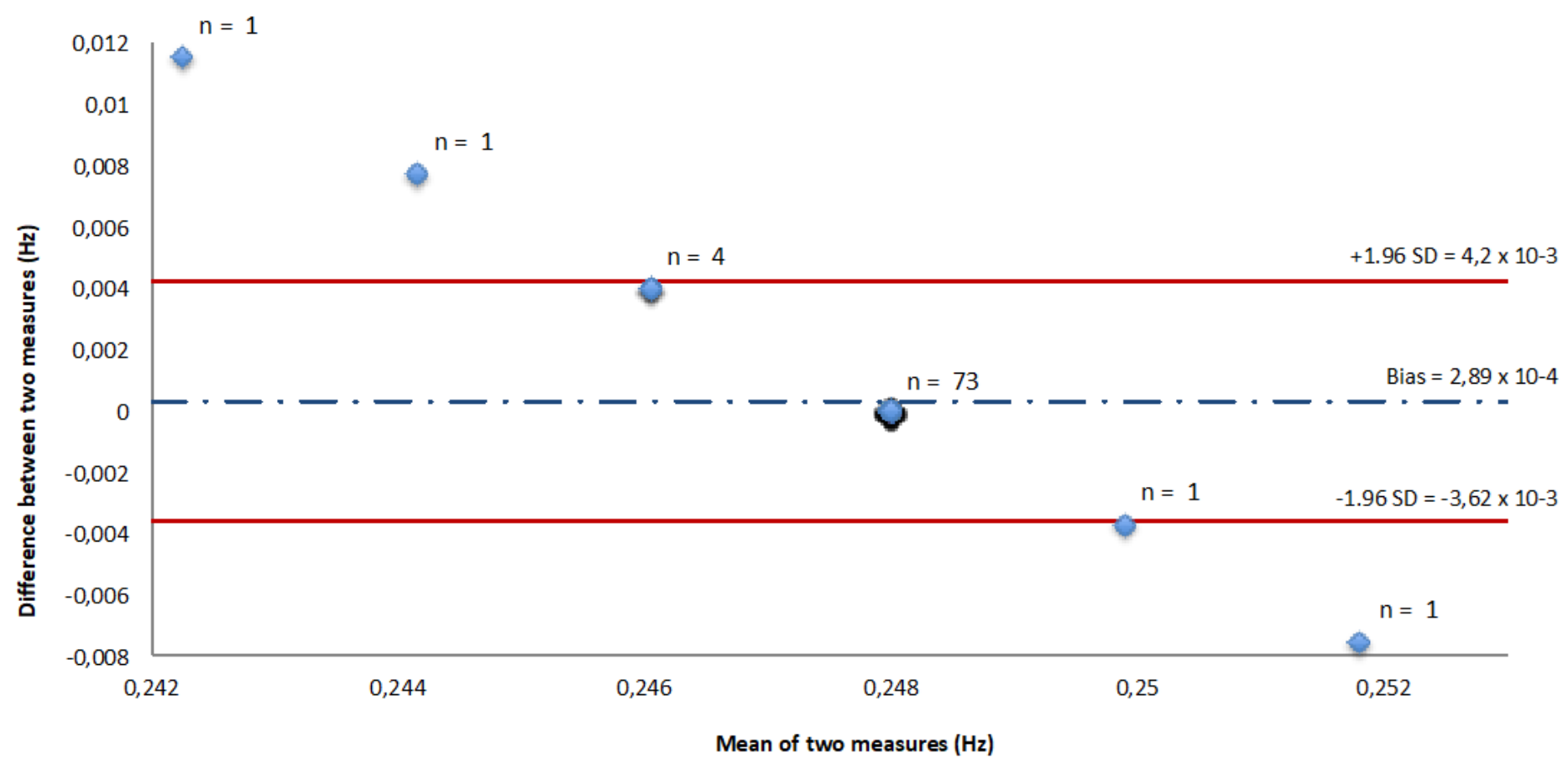

\section{Figure 6}

Plot of Bland-Altman showing bias (broken line) and $95 \%$ limits of agreement (continuous line) on breathing frequency values between ECG and spirometer. 


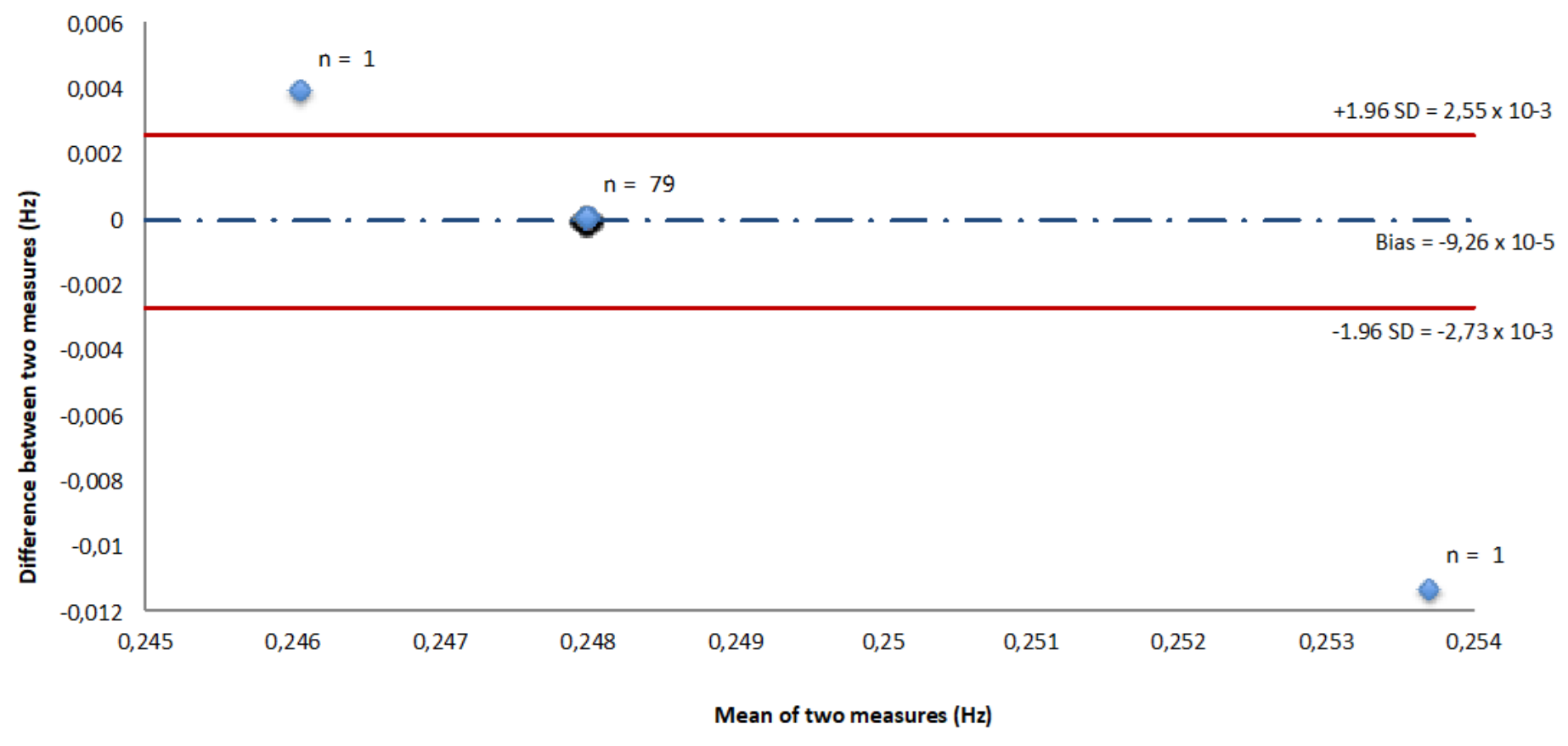

Figure 7

Plot of Bland-Altman showing bias (broken line) and $95 \%$ limits of agreement (continuous line) on breathing frequency values between RR and spirometer. 